EPiC Series in Language and Linguistics
Volume 3, 2018, Pages 124-130
LSP in Multi-disciplinary contexts of
Teaching and Research. Papers from the
16th International AELFE Conference

\title{
El aula intercultural: Análisis contrastivo de las expectativas de alumnos de diferentes nacionalidades ante un curso de IFE. Estudio de un caso
}

\author{
Paloma López Zurita \\ Facultad de Ciencias Sociales y de la Comunicación. Avda. de la Universidad, 4. 11405, Jerez, Cádiz, España
}

\begin{abstract}
Las políticas de movilidad universitaria facilitan la incorporación a las clases de inglés de alumnos de diferentes nacionalidades. El programa de Inglés aplicado a la Publicidad y las Relaciones Públicas de la Universidad de Cádiz ha tenido tradicionalmente al alumnado español como meta. Sin embargo, la incorporación de estudiantes de otros países en el aula la ha configurado como un espacio multicultural donde conviven diferentes concepciones de la realidad que se trasladan al proceso enseñanza-aprendizaje a través de distintas actitudes, conductas o percepciones frente a los mismos temas (Byram, Nichols \& Stevens, 2001). La fusión de estas diferencias crea un espacio multicultural del que los alumnos se pueden enriquecer y aprender, teniendo el inglés como lengua vehicular y motivándolos a percibir la publicidad desde la mirada del otro. El objetivo de este trabajo es identificar sus expectativas según su nacionalidad y formación previa, a través de un cuestionario que identifique sus diferentes niveles, opinión sobre la pertinencia del programa y expectativas ante el curso. El análisis se dará a conocer al propio alumnado, favoreciendo el conocimiento sobre la identidad cultural del resto y su influencia en la visión de la publicidad. Así, se facilita el conocimiento del "otro", modificando, en su caso, los posibles estereotipos y/o prejuicios sobre los distintos grupos culturales y creando un espacio común de convivencia donde el interés por conocer las otras realidades dentro de un marco común de aprendizaje de inglés les sirva de motivación para desarrollar su competencia comunicativa, lingüística e intercultural. Los resultados llevarán a la revisión de los contenidos y metodología de la asignatura, orientándonos hacia un ajuste de los mismos que dé respuesta a las necesidades detectadas, dadas las importantes implicaciones que estas diferentes concepciones tienen en un mundo publicitario global como en el que vivimos hoy en día.
\end{abstract}

Keywords: Multiculturalidad; IFE; Publicidad; Aprendizaje de idiomas

\section{Introducción}

La incorporación a las clases de Inglés para Fines Específicos de alumnos de diferentes nacionalidades es cada vez más habitual debido a las políticas de movilidad universitaria internacional actuales. Si bien tradicionalmente el origen de estos alumnos era europeo, a través del programa Erasmus, en los últimos años su nacionalidad está más diversificada debido a la 
creación de otros tipos de programas, como el Erasmus KA 103, o a través de acuerdos de intercambio bilaterales firmados entre las Universidades implicadas. En el caso de la Universidad de Cádiz, además de alumnos europeos, recibe también estudiantes de Australia, Sudamérica, el Magreb, EE.UU. y antiguas repúblicas soviéticas. Esta situación produce un nuevo escenario de enseñanza-aprendizaje donde no solo conviven diferentes nacionalidades, con lenguas que expresan distintas concepciones del mundo y sistemas de valores (UNESCO, 2007:13), sino que también nos encontramos ante niveles de inicio desiguales, tanto en inglés como en el conocimiento específico de los contenidos en Publicidad y Relaciones Públicas, ya que las carreras de origen de estos estudiantes extranjeros son muy heterogéneas. La asignatura Inglés aplicado a la Publicidad y las RR.PP. (perteneciente al Grado del mismo nombre) de la Universidad de Cádiz ha estado enfocada tradicionalmente al alumno español y su programa se ha diseñado de acuerdo al análisis de sus necesidades concretas. Con la incorporación de alumnos de otros países, el aula se configura como un espacio con distintas actitudes, conductas o percepciones frente a los mismos temas, un espacio intercultural, donde se produce "la presencia e interacción equitativa de diversas culturas y la posibilidad de generar expresiones culturales compartidas, adquiridas por medio del diálogo y de una actitud de respeto mutuo" (UNESCO, 2005:5), que requiere una revisión de sus contenidos y metodología. Esta situación suscita gran interés entre los investigadores en los últimos años (Byram, 1997; Jalo \& Giocchino, 2011; Cuestas, Zamuner \& Peralta, 2013), también en el ámbito de IFE (Alonso \& González, 2008; Guillén-Nieto, Marimón \& Vargas, 2009; Bocanegra, 2015).

La fusión de estas diferencias crea un espacio multicultural del que todos alumnos se pueden enriquecer, teniendo el inglés como lengua vehicular y, en el caso concreto que nos ocupa, aprendiendo a percibir el uso en la publicidad internacional desde la mirada del otro.

\section{Objetivos y Metodología}

El objetivo de este trabajo es identificar las expectativas y opinión del alumnado ante un curso de Inglés aplicado, según su nacionalidad y formación previa, a través de un cuestionario que determine sus diferentes niveles, formas de adquirir la lengua y practicarla y visión sobre la pertinencia del programa y su metodología. Se intenta así facilitar la comprensión de las diferentes identidades culturales que conviven en el aula y su posible distinta visión del mundo publicitario, favoreciendo el conocimiento personal del grupo e intentando modificar, en su caso, los posibles estereotipos y/o prejuicios sobre los distintos grupos culturales. Nuestra finalidad es crear un espacio común de convivencia donde el interés por conocer las otras visiones de la realidad dentro de entorno de aprendizaje de inglés les sirva de motivación para desarrollar su competencia comunicativa, lingüística, intercultural y específica de la materia. En este sentido, coincidimos con Alonso (2006: 17) en que la diversidad idiomática tiene como resultado que:

El hombre siente la necesidad y la curiosidad de comunicarse con congéneres que han adquirido una lengua materna distinta, con los que, por tanto, es incapaz de satisfacer su objetivo. Para superar esta inconveniencia debe aprender una lengua diferente a la materna que coincida con la de su interlocutor o ambos aprender una tercera lengua en la que puedan entenderse.

Los resultados llevarán a la revisión de los contenidos y metodología de la asignatura, orientándonos, en caso de ser necesario, hacia un ajuste de los mismos que dé respuesta a las preferencias detectadas.

El curso está formado por 30 estudiantes, de los cuáles 10 son extranjeros (2 griegos, 2 mejicanos, 3 rusos, 2 kazajos y 1 turcomano); es decir, un tercio de la clase no es española. Las respuestas recibidas fueron 20,12 correspondientes a alumnos españoles (Grupo A) y 8 a 
extranjeros (Grupo B). La encuesta era anónima y constaba de 36 preguntas distribuidas en 4 apartados: a) Datos personales; b) Conocimiento de inglés; c) Uso del inglés y d) Análisis del contenido de la asignatura en cuestión.

Por razones metodológicas y el espacio requerido en el presente trabajo, no se pueden recoger de manera pormenorizada las respuestas a todas las preguntas del cuestionario, por lo que a continuación se exponen resumidos los datos más relevantes de cada apartado junto a las conclusiones parciales a las que nos llevan:

\section{Resultados y Conclusiones parciales}

\subsection{Datos personales}

Ambos grupos están formados por jóvenes cuyas edades, salvo contadas excepciones, oscilan entre los 21 y los 24 años. La carrera de origen es un factor distintivo de cada Grupo: mientras que A pertenece al Grado en Publicidad y RR.PP. o al Doble Grado entre este y Marketing e Investigación de Mercados, en $\mathrm{B}$ encontramos carreras de diversas ramas: Derecho Internacional, Economía, Lingüística y Comunicación Internacional, Marketing, Marketing y Comunicación, Relaciones Internacionales y Relaciones Públicas y Traducción. Como se observa, el conocimiento que estos alumnos extranjeros pueden tener del idioma específico del campo que nos ocupa se puede suponer mucho más limitado que el de los alumnos españoles, pues aún en el caso de un alumno que también estudia Publicidad, su enfoque es distinto al estar dirigido hacia la traducción. Se incrementa así una de las cuestiones problemáticas que, entre otras, pueden surgir en un curso de IFE, esto es, "superar la distancia que separa el conocimiento del alumno de una materia especializada" (Bocanegra, 2011:32). Las diferentes carreras de origen suponen distintos grados de conocimiento del campo de especialización de la asignatura que pueden llegar a interferir en el ritmo de la clase, al tener que explicar conceptos desconocidos para unos y básicos para otros.

En cuanto a la formación previa en idiomas, la diferencia entre ambos grupos es notable. Mientras que en A solo aparece inglés como segunda lengua, en $\mathrm{B}$, con la excepción de los alumnos mejicanos, que comparten la situación de los españoles, todos tienen, además de su lengua materna, conocimientos de inglés, español y, como mínimo, una lengua más (francés, alemán, italiano o ruso). El conocimiento de varias lenguas por parte de B implica que el hábito, y por tanto, la capacidad de aprender idiomas, está más desarrollada que en A.

\subsection{Conocimientos de Inglés.}

En A todos los alumnos tienen el mismo modo de acceso, Bachillerato, por lo que han estudiado inglés durante 12 años. El 83'3\% (10) tiene acreditado su nivel, que es B2 en 4 casos y B1 o equivalente en el resto. En B los años de estudio de este idioma oscilan entre 5 y 8 , con dos excepciones. Solo 1 alumno no posee acreditación de nivel; el resto cuenta con un $\mathrm{C} 1$ e incluso con un $\mathrm{C} 2$ en 1 caso. El hecho de que los españoles hayan estudiado inglés durante más años no tiene como resultado que tengan un nivel más alto, al menos, acreditado. Esta situación nos debería hacer reflexionar sobre la enseñanza de idiomas en nuestro país. Si, pese a estar estudiando más años, nuestros alumnos tienen un nivel inferior al de los extranjeros, puede que estemos haciendo algo incorrectamente. Quizás la respuesta a esta situación venga dada parcialmente a través de las preguntas de este apartado sobre las estancias en el extranjero, su duración y propósito: De los 12 alumnos españoles, 8 sí han estado previamente en un país de habla inglesa, pero la duración de sus estancias suele ser muy breve, alrededor de una semana y 
por motivos turísticos. Por el contrario, en B todos los alumnos salvo 1 han pasado estancias más largas, que van desde 1 mes como mínimo hasta dos años y medio. La razón del viaje en B es también más variada: motivos turísticos (1), laborales (1), haciendo cursos de inglés (2) y, curiosamente, visitando amigos y familiares (4). Ello implica que su contacto con el idioma meta ha sido mayor y en un contexto de inmersión. Parece, pues, que las salidas al extranjero son algo más habitual en $\mathrm{B}$, con lo que su relación con los idiomas se estima mayor y su uso está más naturalizado, familiarizado e independiente del contexto académico.

\subsection{Uso del Inglés}

Ante la pregunta ¿Para qué usas inglés en tu vida diaria? la respuesta mayoritaria en A es "Para asistir a clase". Le siguen en este orden "Para ver la TV, escuchar la radio o programas en internet"; "Para comunicarme personalmente con amigos"; "Para leer periódicos, revistas o textos que me interesen"; "Para comunicarme con amigos online" y, finalmente, "Para redactar trabajos/artículos".

Estos porcentajes varían considerablemente en B, que además de "Para asistir a clase" y "Para leer periódicos, revistas o textos que me interesen" destacan en sus respuestas "Para comunicarme personalmente con amigos"; "Para ver la TV, escuchar la radio o programas en internet" y "Para comunicarme con amigos online". Las respuestas apuntan a que A emplea menos el inglés en su vida diaria, pues 4 alumnos eligen solo 2 de las 6 opciones posibles, otros 4 eligen solo 3, solo 1 elige 4 y también solo 1 elige las 6, mientras que en B 1 alumno marca las 6 opciones posibles, 2 marcan 5, 4 marcan 4 y solo 1 marca 2. Los datos anteriores evidencian que el uso del inglés en la vida diaria del grupo B es mayor que la del grupo A y de un matiz más comunicativo, ya que las opciones elegidas en primer lugar por A se contextualizan en un entorno académico o son de carácter receptivo.

Se les pidió que calificaran su nivel de inglés. En A solo 1 alumno lo considera avanzado, 9 intermedio y 2 inicial. Por el contrario, en B el 50\% lo considera avanzado y el otro $50 \%$, intermedio. B cuenta, pues, con un nivel superior o se siente más consciente de ello. En ambos grupos una amplia mayoría había tenido contacto con el inglés en los tres últimos años; sin embargo, la forma en la que ese contacto había tenido lugar varía considerablemente: En A 9 alumnos mencionan como tal la asistencia a una academia de inglés, para 5 de ellos, el único existente. El resto lo completan viajando al extranjero, hablando con amigos, viendo contenidos en la TV o en Internet. Curiosamente, la academia no se menciona en ninguna de las respuestas abiertas de B, que simplemente contestan "hablando" en 5 casos, o "en clase", "trabajando" o "leyendo". El hecho de que la mayoría de los españoles haya tenido contacto con el inglés en los últimos 3 años solo a través de una academia lo configura en un entorno casi exclusivamente académico, mientras que en B su uso, de nuevo, es más cotidiano y natural, con una forma de practicar mucho más comunicativa y real.

Asimismo, se les preguntó las razones de elegir esta asignatura optativa. Ambos grupos coinciden en la importancia del inglés para su futuro profesional y la mejora que supone en su $\mathrm{CV}$. Sin embargo, en B, algunos alumnos también añaden que no tenían otra opción y que han tenido que optar por esta asignatura obligatoriamente para completar los créditos de su programa de intercambio, pudiendo afectar negativamente a su motivación, que en este caso es mayor en A.

En cuanto al grado de importancia de las destrezas lingüísticas, los resultados, de mayor a menor, en A fueron: 1. Hablar; 2. Entender; 3. Leer y 4. Escribir. En B el resultado fue: 1. Hablar; 2. Entender; 3. Escribir y 4. Leer. Ambos grupos consideran más importantes las destrezas orales. Sin embargo, A parece concebir la comunicación en inglés como un proceso 
algo más receptivo que productivo, ya que apenas lo emplea para escribir y considera la comunicación verbal como la más difícil. Otra diferencia es que en B 6 de los 8 alumnos marcan todas las destrezas como importantes o muy importantes. Este hecho constata que en B conciben el idioma de un modo global, aplicable a cualquier contexto sin discriminar su modo de comunicación.

Ante la cuestión sobre la dificultad que encontraban ante diferentes aspectos del inglés, A le otorga una dificultad media-alta ( $\geq 3$ sobre 5) a "Comunicar mensajes oralmente"; "Redactar textos coherentes"; "Pronunciar bien" y "Usar el vocabulario específico de Publicidad y RR.PP." Concede una dificultad media (3) a "Escribir oraciones gramaticalmente correctas" y "Leer comprendiendo". Finalmente, califica de dificultad media-baja $(\leq 3)$ a "Escribir con corrección ortográfica". Es decir, precisamente lo que marcaron como menos importante es lo que consideran más fácil. En B la mayoría no considera ningún aspecto de dificultad media-alta; entre los de dificultad media señala "Pronunciar bien" y "Usar el vocabulario específico de Publicidad y RR.PP"; de dificultad media-baja incluye "Redactar textos coherentes"; "Escribir oraciones gramaticalmente correctas" y "Escribir con corrección ortográfica" y, finalmente, de dificultad baja o nula "Comunicar mensajes oralmente" y "Leer comprendiendo". Como se observa, ambos grupos prefieren los ejercicios prácticos, aunque A es más crítico o escéptico ante cualquier actividad, ya que en general valora todas más negativamente que B. Se deduce que el trabajo individual y el auto-aprendizaje están más valorados en B que en A.

Sobre cómo les gusta trabajar en clase, A prefiere el trabajo en pequeños grupos o con el grupo entero. El resto de opciones (individual, en parejas o en el laboratorio) son minoritarias. Sin embargo, B prefiere trabajar bien individualmente bien en el gran grupo, dejando la opción preferida por A en último lugar. De nuevo, se constata la tendencia de A a trabajar en grupo y de $\mathrm{B}$ al autoaprendizaje o trabajo individual.

Preguntados también sobre cómo creen que obtienen mejores resultados de aprendizaje en el aula, A suele puntuar todas las opciones más negativamente que B. Lo que consideran menos productivo es: "Escuchar, tomar apuntes y memorizar", "Buscar la información por mí mismo" y "Practicar en un laboratorio". Como se observa, son acciones tradicionales e individuales. Sus actividades favoritas son: "Realizar ejercicios y actividades", "Leer, escribir, hablar y escuchar, reflexionando sobre ello" y "Leer, escribir, hablar y escuchar cuanto más mejor sin detenerme demasiado". Es decir, ven más productivas y eficaces las actividades prácticas. El grupo B es en general más positivo en sus respuestas. Coincide con A tanto en las más valoradas como en las menos, pero incluye entre las primeras "Buscar la información por mí mismo" y da más importancia a las acciones reflexivas que a la cantidad de estas, dato que aparece a la inversa en A. Vuelve a reiterarse la tendencia de B hacia el trabajo individual y el auto-aprendizaje, pues lo valoran mejor que $\mathrm{A}$.

La forma de estudiar la asignatura también es muy similar en ambos grupos: La mayoría prefiere estudiar la asignatura semanalmente y solo un pequeño porcentaje lo deja para unas semanas antes del examen. El tiempo que le dedican es igualmente el mismo en ambos casos, con una media de 2 a 3 horas semanales, empleadas en A mayoritariamente en preparar el trabajo diario para la clase siguiente y en B en realizar los trabajos obligatorios de la asignatura.

La pregunta 13 versaba directamente sobre cómo influye la existencia de alumnado de diferentes nacionalidades en el aula. Las opciones más elegidas, en orden descendiente en A son: Promueve actitudes, conductas y cambios sociales positivos hacia las otras culturas y Crea un espacio común de convivencia agradable del que aprendo (11alumnos); Favorece el conocimiento del otro y modifica los prejuicios sobre los distintos grupos culturales ( 9 alumnos); Ayuda a trabajar en un contexto no excluyente: Todas las culturas en un mismo plano y Modifica estereotipos preconcebidos sobre otras culturas (7 alumnos); Ayuda a descubrir que un 
conocimiento tiene el mismo valor que el otro y Ayuda a conocer mejor mi propia cultura (1 alumno). Solo hay un caso que marca algo negativo: Me siento más cohibido para participar en clase expresando mis opiniones.

En orden en B es: Favorece el conocimiento del otro y modifica los prejuicios sobre los distintos grupos culturales y Modifica estereotipos preconcebidos sobre otras culturas (5 alumnos), Promueve actitudes, conductas y cambios sociales positivos hacia las otras culturas (4 alumnos) y Ayuda a trabajar en un contexto no excluyente (3 alumnos). Curiosamente, la elección preferida en A no es de las más elegidas en B. A valora esta existencia como algo muy positivo y agradable para aprender, mientras que B no percibe la situación como una oportunidad especial de aprendizaje de inglés.

\subsection{Contenidos de la asignatura}

Los alumnos españoles creen que tendrán una dificultad media o alta para superar la asignatura. Entre los alumnos extranjeros destaca que tendrán poca dificultad (50\%) e incluso hay quien señala "ninguna". En cuanto al análisis de los contenidos de la asignatura los alumnos en A valoraron más positivamente aquellas unidades totalmente específicas de su especialidad, esto es en orden de preferencia: "Sales Techniques" y "Advertising"; en segundo lugar prefieren aquellas que, aun siendo específicas, tienen contenidos más generales aplicados a la Publicidad: "Branding"; "The Language of Meetings. Oral Presentations"; "Market Strategy" e "International Business Styles, Cultural Awareness". Las menos valoradas (aunque siempre por encima de 3) son aquellas más relacionadas con los aspectos menos creativos de la Publicidad, enfocados al mundo de los negocios, como "Innovation", "Competition and the Market" o "Quality control". En este caso, la diferencia entre los dos grupos es la más marcada. El orden obtenido en B es precisamente el inverso. Prefieren las unidades más genéricas, que les suponen menos problemas de comprensión de su lenguaje específico, y sobre todas destaca la unidad sobre temas culturales y sus implicaciones sociales y publicitarias. Por esta misma razón, ante la pregunta sobre la relación de estos contenidos con el Grado al que pertenecen, mientras que A los estima relacionados $(83,3 \%)$ o muy relacionados $(16,7 \%)$, B opina en un $50 \%$ que no lo sabe, ya que realmente desconocen las demás asignaturas como para poder establecer esta comparación.

\section{Conclusiones}

La interculturalidad en el aula, como se ha visto en las conclusiones parciales del apartado anterior, es muy positiva para romper barreras y aprender de los demás desde un punto de vista sociológico o cultural. Para aprender inglés también, pero sobre todo para los alumnos españoles, que valoran esta situación como una oportunidad especial de practicar, ya que no tienen tantas oportunidades de hacerlo y suelen relacionar el estudio de idiomas con contextos casi exclusivamente académicos, de ahí su cierto escepticismo hacia las actividades que se realizan en el aula y su valoración altamente positiva de la inclusión en la misma de alumnos de otras nacionalidades. El reto del profesor está en ser capaz de mantener el interés por unos contenidos que no son de su especialidad en un alumnado extranjero y en motivar al español a ver la lengua como un vehículo para traspasar fronteras y conocer mundo, más allá de su aprendizaje específico. Cómo hacerlo, por razones de espacio, tendrá que ser el objetivo de futuras publicaciones. 


\section{References}

Alonso, A. (2006). La competencia intercultural en la enseñanza del inglés dentro del contexto turístico. Encuentro, $16,17-26$.

Alonso, A. \& González, L. (2008). El desarrollo de la competencia intercultural como clave en la adquisición del inglés dentro del contexto especifico del turismo. En L. Pérez, I. Pizarro \& E. González-Cascos Ccoords.), Estudios de metodología de la lengua inglesa (IV). (pp. 187-200). Valladolid: Universidad de Valladolid.

Bocanegra, A. (2012). El profesor de Inglés para Fines específicos ante el espacio Europeo de Educación Superior. Aula, 18, 29-41.

Bocanegra, A. (2015). La competencia intercultural en los manuales de texto con fines específicos a través de la evaluación de sus indicadores y niveles de dominio. Cuadernos de Filología Francesa, 26, 29-43.

Byram, M. (1997). Teaching and Assessing Intercultural Communicative Competence. Clevedon: Multilingual Matters Ltd.

Byram, M., Nichols, A. \& y Stevens, D. (2001). Developing Intercultural Competence in Practice. Clevedon: Multilingual Matters Ltd.

Cuestas, A. D; Zamuner, A \& Peralta, S. (2013). Un permanente desafío en el ámbito del inglés con fines específicos: El desarrollo de la competencia comunicativa intercultural. Puertas Abiertas (9). En Memoria Académica. Disponible en: http://www.memoria.fahce.unlp.edu.ar/art_revistas/pr.6285/pr.6285.pdf

Jalo, M. \& Ciocchini, C. (2011). La Educación global en la clase de inglés: Interculturalidad y pensamiento crítico.

Puertas Abiertas (7). En Memoria Académica. Disponible

http://www.memoria.fahce.unlp.edu.ar/art_revistas/pr.5734/pr.5734.pdf

Guillén-Nieto, V., Marimón, C. \& Vargas, C. (2009). Intercultural Business Communication and Simulation and Gaming Methodology. Bern: Peter Lang.

UNESCO (2005). Convención sobre la protección y promoción de la diversidad de las expresiones culturales. París: UNESCO.

UNESCO (2007). Directrices de la UNESCO sobre la Educación Intercultural. París: UNESCO. 